\title{
Universal Grammar and the Basic Variety
}

\author{
Manfred Bierwisch Humboldt-Universität Berlin
}

\begin{abstract}
The Basic Variety (BV) as conceived by Klein and Perdue $(K \& P)$ is a relatively stable state in the process of spontaneous (adult) second language acquisition, characterized by a small set of phrasal, semantic and pragmatic principles. These principles are derived by inductive generalization from a fairly large body of data. They are considered by $K \& P$ as roughly equivalent to those of U niversal Grammar (U G) in the sense of Chomsky's M inimalist Program, with the proviso that the BV allows for only weak (or unmarked) formal features. The present article first discusses the viability of the BV principles proposed by $K \& P$, arguing that some of them are in need of clarification with learner varieties, and that they are, in any case, not likely to be part of UG, as they exclude phenomena (e.g., so-called psych verbs) that cannot be ruled out even from the core of natural language. The article also considers the proposal that learner varieties of the BV type are completely unmarked instantiations of U G. Putting aside problems arising from the $M$ inimalist Program, especially the question whether a grammar with only weak features would be a factual possibility and what it would look like, it is argued that the BV as characterized by $K \& P$ must be considered as the result of a process that crucially differs from first language acquisition as furnished by $U \mathrm{G}$ for a number of reasons, including properties of the BV itself. A s a matter of fact, several of the properties claimed for the $B V$ by $K \& P$ are more likely the result of general learning strategies than of language-specific principles. If this is correct, the characterization of the $B V$ is a fairly interesting result, albeit of a rather different type than $K \& P$ suggest.
\end{abstract}

\section{Introduction}

B ased on systematic data from an extensive study of spontaneous second language acquisition, $\mathrm{K}$ lein and Perdue (henceforth $K \& P$ ) develop the notion of the Basic Variety as a specification of general properties the more or less stable state resulting from adult language acquisition tends to exhibit. The structure assumed for the $B$ asic Variety (BV for short) is presented as the result of empirical analysis and inductive generalization over a wide variety of speakers with different combinations of first and second (source

A ddress for correspondence:A rbeitsgruppe 'Strukturelle G rammatik',H umboldt-U niversität B erlin, J ägerstrasse 10-11, D -10117 B erlin, G ermany. 
and target) language. A ccording to $K \& P$, the result of adult $L 2$ acquisition can be characterized by a small set of syntactic, semantic and pragmatic principles, together with some general conditions on the organization of lexical knowledge.

Two points about this rather strict empiricist orientation seem to me worth noticing from the outset. First, it differs from a great deal of $L 2$ research by taking the leading question to be 'How do learners go about formulating what they want to express?' instead of 'H ow far do they approximate the structure of the target language?'. In other words, $K \& P$ attempt to assess the learner variety essentially in its own right, rather than under the auspices of a putative norm to comply with. This does not deny, of course, that learners depend on the language of their environment, but it takes as basic the intention to organize and convey messages rather than to attain a given language. This formulation might oversimplify matters a bit, but it correctly indicates the perspective $K \& P$ pursue, I guess. Second, and perhaps even more important, $K \& P$ consider the principles they arrive at by inductive generalization as not merely an incidental collection of possibilities that could just as well be otherwise, but as an essential pattern indicative of basic conditions of a rather general character. In the following discussion I will take this orientation as decisive, because it is if and only if the structure of the BV has the principled status claimed by $K \& P$, that the more interesting questions relating the $B V$ to basic conditions of linguistic knowledge arise in the first place.

Now, most of the assumptions embodied in this characterization of the BV appear to be fairly plausible and not too surprising, after all. A nd plausible in a fairly straightforward sense is in fact what they are intended to be. However, the proposals by means of which the BV is specified are by no means derived merely by induction over empirical data; $K \& P$ rather rely on theoretical constructs such as topic, focus, control (a concept to which we will return), NP, or subject, and on assumptions of essentially hypothetical character like the conjecture that (all and only) N Ps have referents or that the given-new contrast is to be distinguished from topic-focus-articulation. It is this hypothetical aspect of the BV that raises the most interesting questions, especially as $K \& P$ relate it to the notion of $U$ niversal Grammar in the sense developed in the tradition of generative linguistics.

The following discussion has two parts. First I will make a number of remarks concerning the descriptive aspect of the BV, and the particular assumptions specifying its content and organization. I will 
then discuss the assumptions about the status of the BV and its relation to U niversal G rammar.

\section{O $\mathrm{n}$ the structure of the B asic Variety}

The BV is essentially characterized by three types of constraints and certain principles determining the representation of time and space. $K \& P$ discuss the following characteristic, but possibly not exhaustive principles.

1) a. phrasal (i.e., syntactic) constraints $\mathrm{PH}$ 1-PH 3

b. semantic constraints SE M 1-SE M 3

c. pragmatic constraint PR 1

2) Principles I-III on expression of temporality

Furthermore, they mention certain observations concerning the lexical repertoire of the $B V$ :

3) a. The lexical repertoire of the Basic Variety consists almost exclusively of morphologically simple, invariant forms,

b. The lexical repertoire depends on that of source language $S L$ and target language $T L$, with strong priority of $T L$,

c. The lexical repertoire contains almost only major lexical category items, and virtually no functional elements.

Lexical items are supposed to be made up from phonological, syntactic and semantic features according to usual assumptions. I will return to some consequences to be drawn from this conjecture.

The BV could presumably be characterized also by certain phonological constraints (depending more heavily on properties of $\mathrm{SL}$ than the syntactic and semantic principles), an aspect $K \& P$ are not concerned with.

Let me first turn to some comments on the phrasal constraints. A ctually, $K \& P$ list eight variants altogether, distinguishing e.g., $N P_{1}-V-N P_{2}$ from N $P_{1}-C o p-N P_{2}$, where Cop represents the copula. If items of category $V$ and $C o p$ are, however, specified by grammatical features, as assumed by $K \& P$, then these constraints could be collapsed, relying only on different feature specifications of V. Furthermore, PP is accounted for in $K \& P^{\prime}$ 's constraints only as a complement of Cop, while they provide examples clearly showing it as a complement or adjunct of other verbs as well. M ore generally, all the differences captured by the (sub) constraints $\mathrm{PH}$ la, $\mathrm{PH} 1 \mathrm{~b}, \mathrm{PH} 1 \mathrm{c}$ and $\mathrm{PH} 2$ could be reduced to one constraint which relies on the lexical properties of the verbal head.A s PH 3 only adds 
cases where the subject is not overtly realized, presumably as an optional variant depending on conditions $\mathrm{PH} 3$ does not specify anyway, ${ }^{1}$ this could be included into one overall constraint of the following sort:

4) (NP)-V-(XP-(XP)) where $X=N$, $A$ or $P$, depending on the subcategorization of $\mathrm{V}$.

In the light of proposals on 'Bare Phrase Structure' in Chomsky (1995), a framework K\& P refer to in other respects, (4) could be further reduced to the operation Merge given the lexical characterization of the head. ${ }^{2}$

While these issues might be considered primarily as matters of elegance and parsimony of formulation (but see below for comments on problems of completeness), the question whether and in which way linear ordering is to be represented in phrasal constraints turns on more substantial issues. The claims $K \& P$ want to defend in this respect are not quite clear. On the one hand, they acknowledge some variation in verb placement, essentially due to properties of the source language (p. 314, n. 16); on the other hand, verb second seems to be taken as fundamental for the BV, provided that cases of subject deletion are accounted for according to $\mathrm{PH} 3$. If this assumption would correctly account for the result of acquisition with respect to different target language structures, it would have non-trivial consequences for the more general claims to be discussed below, because verb second would be a property of U niversal Grammar that allows for deviations only on special demand.

A somewhat different problem is related to the following general characterization, which concludes $K \& P^{\prime} s$ summary of $B V$ principles:

IV. Strikingly absent from the Basic Variety are (a) free or bound morphemes with purely grammatical function, and (b) complex hierarchical structures, in particular subordination. (p. 332)

A s to IV a, its validity is difficult to assess in view of determiners or even relative pronouns that appear to figure in the BV (see [22]

\footnotetext{
${ }^{1}$ In their note $18, \mathrm{~K} \& \mathrm{P}$ rely on $\mathrm{PH} 3$ for alternations like Charlie arrive vs. arrive Charlie, which would belong to 'unaccusative constructions' rather than subject deletion. But that does not affect the present discussion.

${ }^{2}$ It is not quite clear whether $K \& P$ simply skip the discussion of the structure of $N P, P$ and A dj, taking this to be a trivial issue, or whether the BV is supposed to take NP as one of the options listed on page 319 (proper name, (D et) noun, pronoun), with PP presumably coming out as P NP. Either way, the syntax of the BV seems to be in need of further elaboration, a problem to which I will return immediately.
} 
below $\left.{ }^{3}\right)$. A s to (b), I don't think that it can be maintained in any serious sense. While $K \& P$ on the one hand incorporate IV $b$ in the principles $\mathrm{PH} 1$ to $\mathrm{PH}$,as these - if taken literally - would not allow for any recursion (but see note 2), they on the other hand provide examples which clearly require complex NPs and various types of subordination, as shown by the following cases:

16] [ame] le chapeau avec le tabouret (ZM F)

22] [se] la dame QUI a volé le pain (GFS) ${ }^{4}$

It is not only phenomena like these which highlight a more general problem about the $\mathrm{BV}$ : is the outline $\mathrm{K} \& \mathrm{P}$ present to be understood as essentially complete with respect to the aspects the principles belong to? Or is the presentation a more or less representative fragment that might be completed as research goes on? This alternative has important consequences not merely for the descriptive coverage of the $B V$ as presented by $K \& P$, but notably for the ontological status to be ascribed to the BV, a point to be taken up in section III. It seems to me that $K \& P$ intend their presentation to be complete in the sense that minor adjustments might be indicated, but the essential characterization of the BV does not require or even allow for substantial amendments. In any case, I will interpret the BV in this sense, as it is only this construal which provides an interesting perspective on the relation between the $B V$ and the language capacity $K \& P$ are interested in. This does not exclude, however, that further aspects could be added, characterizing, e.g., additional patterns of conceptual organization in the vocabulary, or - as already mentioned - principles of phonological structure. It only takes those aspects that are explicitly covered to be essentially complete. In other words, something like $\mathrm{PH} 1$ to $\mathrm{PH} 3$ - or (4), for that matter - together with the relevant lexical information must be taken to specify completely the syntax of the BV. This has crucial consequences for the discussion in Section III, and it also raises non-trivial questions as to which lexical information is available, and in which format.

$L$ et us turn next to what $K \& P$ call semantic constraints, dealing essentially with thematic roles and their mapping on phrasal positions. SEM 1 and SEM 2 are based on the concept of control,

\footnotetext{
${ }^{3} \mathrm{~N}$ umbers in brackets [ ] are from $K \& P$, those in parentheses ( ) are my own numbers.

${ }^{4} \mathrm{~A} S \mathrm{~K} \& \mathrm{P}$ provide not much more than a dozen examples altogether, the fact that at least two require some sort of subordination seriously challenges the validity of IV b. In a more general sense, I would raise strong doubts with respect to the claim that the language of L 2 learners should not contain subordination, or recursive structures, in any reasonable way.
} 
which is, according to $K \& P$, a graded notion ranging from strong control of one participant over the other, as in hit, break, to complete absence of control in cases like be, resemble. ${ }^{5}$ W hat SE M 1 should state is, of course, not that the referent with highest control comes first, but something like

SEM 1' The NP the referent of which has highest control comes first

provided that control obtains. If I understand SEM $1^{\prime}$ and its consequences right, the BV would exclude constructions like

5) a. The proposal pleases J ohn

b. The book reminds me of my childhood

c. The car belongs to Mary

d. The argument convinced Bill

6) a. Der Versuch glückt mir (I succeed with the attempt)

b. Das Haus fällt jedem auf (Everybody notices the house)

c. Seine Kleidung täuscht dich ( $\mathrm{H}$ is dressing deceives you)

I wonder whether this claim is borne out, i.e. whether the BV does not allow so-called psych verbs, as in (5a) and (5b). If this is correct, this would have consequences for the status of the BV to be discussed below.

While SEM 1' raises an empirical issue with respect to learner varieties, we now turn to problems of a more principled, conceptual type:

SE M 2 Controller of source state outweighs controller of target state

This principle is meant to account for cases like [7], where the referents of NPs participate in two subsequent states:

7] Charlie give present for young children (SIE)

If one adopts the notion of control in the sense of $K \& P$, it makes sense to assume that Charlie has control over the present in the source state and the children have control over it in the target state,

${ }^{5} \mathrm{~K} \& \mathrm{P}$ refer to Comrie (1981), a more recent account would presumably be D owty's (1991) notion of Protoroles, defining agentivity in a similar vein. I will not discuss the foundation of thematic roles more extensively in this connection, but I want to express my doubt that lexical items can be classified along these lines in sufficiently general ways for reasons that will become obvious shortly. 
where the former determines the ordering of the referring N Ps. B ut now consider cases like (8), where the change of possession has the opposite direction with Charlie being the controller in the target state:

8) Charlie take present from young children

H ence either control must be redefined in unclear ways, or SE M 2 simply does not hold. Things are further complicated if one takes into account cases like (9), where besides the theme denoted by the NP the car, the money for which it is bought is involved, with exchange of control being definitional:

9) Charlie bought the car from $\mathrm{H}$ arry (for $\$ 500$ )

A nd even if one would ignore the financial equivalent, SE M 2 would be violated just as in (8), as Charlie is in control over the car in the target state, rather than in the source state, and yet Charlie precedes $\mathrm{H}$ arry. Notice that buy constitutes a systematic group of verbs, together with sell, rent, borrow, lease, order, receive describing exchange of use or possession, which clearly belong to the core vocabulary and must not be excluded from the BV.

$K \& P$ suggest furthermore that SE M 2 can be extended to verbs of saying, where the 'sayer' comes first, the hearer comes second and the 'said' comes last. But this generalization fails for (10) in exactly the same way as SE M 2 fails for (8):

10) a. John listens to daddy

b. Mary heard the story from her sister

I conclude that SEM 2 is obviously mistaken and must either be substantially reformulated or discarded altogether. ${ }^{6}$ E ither way, given the highly restricted machinery the BV is supposed to rely on, failing on one principle out of three is not a very strong score.

Let me briefly mention SEM3, as this participates in the assignment of thematic roles to syntactic positions:

${ }^{6}$ I would assume that verbs like give and take should be analysed roughly along the following lines, providing the basis for a somewhat different type of mapping from semantic variables to syntactic positions:

/ give / xCAUSE ( ( $x$ HAVE $z$ ) CHANGE-TO ( yHAVE $z$ ))

/ take / x CAUSE ( ( yHAVE $z$ ) CHANGE-TO ( $x$ HAVE $z$ ))

Even though this is nothing but a raw sketch,it indicates that causation,rather than control, would be relevant for the assignment of the semantic variable $x$ to the grammatical function subject in these cases. See Bierwisch (1997) for further discussion of these matters. 
SE M 3 Theme before relatum in target position

While $K \& P$ intend this principle to apply essentially to verbs of motion (which are supposed to include verbs of giving), it can obviously be generalized to verbs of position, as in (11); to prepositions, as in (12); and it is not restricted to target position, but covers source position as well, as shown in (13): ${ }^{7}$

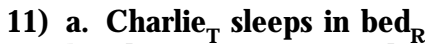

b. The carpet $_{T}$ covers the table $_{R}$

c. L ondon $_{T}$ exceeds $\mathrm{G} \mathrm{lasgow}_{\mathrm{R}}$ in size

12) a. The road to Sacramento ${ }_{R}$
b. A house $_{T}$ at the beach

c. Mary $_{T}$ is in her office ${ }_{R}$

13) a. Charlie $e_{T}$ came from $L_{\text {ondon }}$

b. Charlie left Paris $_{R}$

c. Mary lost her cap in the woods $s_{R}$

d. R egards $\mathrm{S}_{\mathrm{T}}$ from Charlie $_{\mathrm{R}}$

O nce again, however, there seem to be cases that violate SEM 3, even though they cannot easily be dismissed from the BV as marginal:

14) a. The box $x_{R}$ contains three apples

b. The garden swarms with bees

c. Der immel $_{R}$ hängt voller Wolken T $_{T}$ (The sky is full of clouds)

d. u men'a $\mathrm{a}_{\mathrm{R}}$ kniga $_{\mathrm{T}}$ ('with me (is) book' = I have a book)

e. On the table $e_{\mathrm{R}}$ is a book ${ }_{\mathrm{T}}$

A s a matter of fact, as pointed out to me by Chris Wilder, the rather general phenomenon of so-called 'locative inversion', illustrated by (14e), is a widespread and in many languages unmarked construction, which should be expected in learner varieties as well. In other words, SE M 3 as stated by $K \& P$ is at best a generalization stating the normal case within a certain domain; it does not indicate, however, the range, reasons and conditions of possible variation.

It might be added, incidentally, that there are various types of phenomena belonging to the realization of thematic positions for which the BV does not make any options. The principles SE M 1 to SE M 3 do not indicate, for example, how verbs like resemble, differ, constructions like be similar, be related and, more generally,

\footnotetext{
${ }^{7}$ The subscript ${ }_{T}$ and ${ }_{R}$ mark the theme and the relatum, respectively.
} 
adjectives and nouns deal with their arguments or adjuncts. N otice that this gap cannot be accounted for by merely observing that phenomena like these are rare or marginal in learner varieties, because the BV should be complete in the sense that either the exclusion or the accommodation of the phenomena in question must be defined and motivated.

In short, K\&P's semantic principles, expressing essentially the constraints on argument structure embodied in the BV, cannot fully account for the facts they are designed for, leaving unexplained a number of pertinent phenomena. I do not believe that this is simply a matter of adding some more principles SE M 4 to SE M n, but rather an issue of conceptual organization. In much the sense in which phrasal syntax might ultimately derive from just one (or may be two) combinatorial principles ${ }^{8}$ relying on lexical information that must be available anyway, syntactic realization of thematic roles might be determined by only one general operation applying to appropriate argument structure information of lexical items. ${ }^{9}$

Turning next to some remarks on PR 1 , the only constraint that $K \& P$ take to deal with conditions of pragmatics, one might first wonder whether this is meant to meet the Completeness Condition in the sense indicated earlier. The fact that $K \& P$ call it $P R 1$, although there is no other pragmatic constraint, might in fact hint at amendments they have tacitly in mind. In any case, the principle

\section{PR 1 Focus expression last}

clearly must be reformulated or supplemented..$^{10}$ The more general problem here is, however, whether PR 1 or its replacement establishes and exhausts a domain of linguistic knowledge underlying learner varieties, and especially the BV. I will refrain

${ }^{8}$ I should emphasize that the schema (4) is not intended to state such a principle; what I have in mind is rather a more abstract pattern like the functor-argument structure of categorial grammar or the basic operations of B are Phrase Structure considered in Chomsky (1995).

${ }^{9}$ Various proposals have been made along these lines, e.g., by G rimshaw (1990), Jackendoff (1990) or Bierwisch (1997), to mention only a few.

${ }^{10}$ R elying on the rather restricted exemplification provided by $K \& P$, I would nevertheless assume that

8] d. arrive *otra* personne (R SF) $<$ arrive other person>

would have to come out as arrive personne otra, if PR 1 were to apply literally. O nce again, the BV does not deal with the internal organization of N P, even though learners clearly have principles controlling this aspect. A nd I would expect focus stress to allow variation that is in conflict with PR 1 even in arbitrary learner varieties. $K \& P$ furthermore point out - in their Section V.2 - that, if need arises, learners come up with cleft constructions the essential purpose of which would be to overcome PR 1. 
from any further speculation and briefly turn to the treatment of temporality and space as aspects of the BV.

$K \& P^{\prime} s$ selection of space and time out of the range of semantic, conceptual or perceptual domains cannot merely be determined by the empiricist orientation they subscribe to. ${ }^{11}$ Whether the choice reflects a kind of $\mathrm{K}$ antian concern for space and time as basic categories of perception, or just implicit methodology that tries to capture the more general structures projected on the data, can be left open. I merely want to point out that temporality and spatial relations are clearly conceptual domains and the principles ascribed to the expression of these domains could be construed as semantic in a stricter sense than SE M 1-SE M 3, which are rather matters of the semantic-syntax interface.

The treatment of these two domains with respect to the BV is somewhat different. Temporality is structured by three principles, handling the time under discussion (Topic Time TT) and its relation to the time of the reported events (Situation Time TSit):

I TT is initially fixed, either explicitly or by default;

II Change of TT must either be marked or follows the order of mention;

III TT and TSit coincide.

These are clearly conditions of cognitive parsimony, presumably to be extended or refined if need arises, e.g., in case of warnings, promises, expectations, etc. turning on future events. H ence what principles I to III (and their conceivable extensions) are dealing with are conditions on something like cognitive economy. Spatial relations on the other hand are characterized by somewhat sketchy remarks on static and changing localization, where the expression of basic relations is assumed to be determined to a larger degree on the way the target language organizes the expression of space. Whether the difference between the largely language independent structure of temporal organization and the more language dependent expression of spatial relations is an incidental observation, or should be construed as a matter that is characteristic of the BV, is left implicit by $K \& P$. G iven the incomplete character of these exemplifications, the interesting, but clearly incomplete, discussion of space and time should presumably be understood as a characterization of the sort of phenomena to be observed, rather than a systematic proposal concerning the principles (of the

\footnotetext{
${ }^{11}$ It is unclear to me, whether domains of this sort can be considered as inductively established in any reasonable sense. If so, however, one could think of various other candidates of conceptual organization, such as modality, causality, possession, colour, social roles (including kinship), or various aspects in terms of which persons are identified.
} 
semantic component) of the BV.

To sum up, it seems to me that the principles $\mathrm{PH} 1-\mathrm{PH} 3$ determining the phrase structure and the principles SE M 1-SE M 3 governing thematic role assignment should be construed as a preliminary account of the BV, or rather its syntactic component, ${ }^{12}$ where SEM 1-SEM 3 determine the argument structure the BV provides for. Keeping in mind the problems concerning the specific content of these principles, I will now turn to issues of the more general status of the BV.

\section{B asic Variety and U niversal G rammar}

The main point to be discussed in this respect is $K \& P^{\prime} s$ claim that the principles of the BV should be identified with U niversal G rammar (UG), and more specifically with the principles proposed in the M inimalist Program of Chomsky (1995) under the particular option that all formal features are weak, determining a language that is 'perfect' in a rather specific sense. ${ }^{13}$ This claim is interesting and in fact surprising, as there is no reason to expect the empirical generalizations of the $\mathrm{BV}$ to coincide with the theoretical principles explored in the $M$ inimalist Program, let alone a rather specific choice of feature values within that framework. I will first discuss the chances of this particular claim, and then consider the relation of the BV and UG under a slightly more general perspective.

To begin with, U niversal G rammar, or rather the computational system $C_{H L}$ of human language in Chomsky's recent terminology, provides the operations on which the internal or I-language of a speaker/hearer is based. The I-language provides the mapping between two rather different domains of mental structure, viz. the range of articulatory and perceptual patterns $A-P$ and the system of conceptual-intentional organization of experience I-C. ${ }^{14} \mathrm{O}$ this account, an I-language provides a systematic, computable correspondence between sounds (or other signals) and meanings. M ore technically, I-language defines an open set of pairs $\langle\pi, \lambda\rangle$,

\footnotetext{
${ }^{12}$ Whether and in which sense the language capacity must go beyond syntactic principles will be taken up below.

${ }^{13}$ This assumption turns on the distinction between strong and weak formal features made in Chomsky's M inimalist Program. Notice, incidentally, that this distinction is not to be confused with that between marked and unmarked values of features or parameters. In other words, Chomsky's 'perfect' language is not necessarily the most unmarked language.

${ }^{14}$ Chomsky calls A-P and C-I 'performance systems' by means of which I-language is interpreted. This term is somewhat misleading because it might be construed as suggesting that - using the competence-performance distinction of e.g., Chomsky (1965) - conceptual or articulatory systems are merely modes of using language, not based on knowledge or competence of their own.
} 
where $\mathrm{p}$ and $\mathrm{I}$ are interpreted in A-P and C-I, respectively, schematically:

15) $\mathrm{A}-\mathrm{P}<===>\pi-\lambda<===>\mathrm{C}-\mathrm{I}$

$\overline{\text { I-language }}$

$\pi$ and $\lambda$ are configurations of primitive elements that make up representations of Phonetic Form PF and the Logical Form LF, respectively, PF and LF being the interface systems relating Ilanguage to $A-P$ and $C-I$. E ach particular I-language is based on a lexical system $L S$, the members of which are sets of primitive elements, and more specifically triples $\langle\pi, \lambda, \gamma\rangle$, where g consists of formal, i.e. grammatical features with no interpretation outside $\mathrm{C}_{H L}$, while $\pi$ and $\lambda$ define form and meaning of the lexical item. N ow the M inimalist Program is based, among others, on two crucial assumptions:

16) a. Differences between different I-languages are completely determined by $L S$, i.e., the idiosyncratic information contained in the lexical repertoire.

b. Syntactic differences between I-languages follow from the effect that strong or weak formal features of lexical items have on their syntactic position.

Whether some formal feature (like e.g., Case or N umber ${ }^{15}$ ) is strong or weak for a certain type of entry in a given language, must be determined in language acquisition, just as the identification of other features of lexical items is a matter of language acquisition. In other words, there are no grammatical rules, in terms of which two languages might differ. Whatever might be different for two languages $L$ and ' $L$ ' (and hence dependent on experience and learning of some sort) is based on their lexical elements, which then trigger the differences mentioned in (16b). ${ }^{16}$ The way in which these differences are accounted for is roughly as follows.

$\mathrm{C}_{\mathrm{HL}}$ provides an operation Select, which picks up elements from the lexical system LS, an operation M erge, which integrates these elements stepwise into a complex expression, ${ }^{17}$ and an operation

\footnotetext{
${ }^{15}$ Like many other details, the question whether formal features are in fact without conceptual interpretation, and which features can be strong or weak in the relevant sense, is controversial and difficult to decide. Categories like G ender, Number, Person, which are often treated as strong formal features, are clearly susceptible to conceptual interpretation in many cases.

${ }^{16}$ This does not, presumably, include the phonological component, which is in fact based on features selected from a universal inventory, but might still rely on language-specific rules. ${ }^{17}$ It is roughly this operation that specifies the phrase structure and should thus replace $\mathrm{PH} 1$ to $\mathrm{PH} 4$, or (4), for that matter, as indicated above. See note 8.
} 
M ove, which reorders elements so that their formal features can be matched or 'checked'. M ove must be 'overt', if the relevant features are strong; it is 'covert', i.e., without effect in the explicit form in $P F$, if the features are weak. For the sake of illustration, look at the well known differences of finite verb position in German subordinate and main clauses, as opposed to their E nglish counterparts, as shown in (17), which can be accounted for by different strength of formal features of finite verb forms and complementizers like daß, that, etc.

17) a. daß Peter den Ball fängt

(a') *that Peter the ball catches

b. *daß Peter fängt den Ball

(b') that Peter catches the ball

c. *Peter den Ball fängt

$\left(c^{\prime}\right) *$ Peter the ball catches

d. Peter fängt den Ball

(d') Peter catches the ball

The details that account for these and other phenomena of verb placement in G erman and E nglish need not concern us here. ${ }^{18}$ They are in need of clarification anyway, for reasons concerning technical aspects of the framework ${ }^{19}$ as well as its application to concrete facts like (17). L et us assume, nevertheless, that the basic orientation of the $M$ inimalist Program is on the right track, relating it now to $K \& P^{\prime} s$ orientation of the B asic Variety.

$A$ s already mentioned, $K \& P$ suggest that the $B V$ is characterized by properties that would show up on the basis of $\mathrm{C}_{\mathrm{HL}}$ if all formal features appearing in a given I-language are weak. This claim is difficult to assess for two reasons: on the one hand, the M inimalist Program is very much in flux, and it is quite unclear whether an Ilanguage based on only weak features is to be considered as an actual possibility in the first place, once the alternation of strong and weak features is available. ${ }^{20} \mathrm{On}$ the other hand, the actual

\footnotetext{
${ }^{18}$ For a recent proposal, see Wilder (1995), who deals with the fairly complex pattern by means of different values for three features, which do not allow, incidentally, a simple statement as to which language is 'more perfect'.

${ }^{19}$ In a critical discussion of the framework, $G$ ärtner (1997) raises a fair number of technical questions, the clarification of which might not be without consequences even for empirical analyses.

${ }^{20}$ The motivation behind this consideration is highly speculative. But for the sake of argument, consider the $\mathrm{M}$ arkedness Theory of phonetic features. In a way comparable to $K \& P^{\prime}$ 's plea for an I-language with only weak features, one might argue for a 'perfect phonology' which allows only unmarked values of phonetic features - clearly a conjecture that could not possibly be realized.N ote that I'm not claiming that the logic of weak features is that of unmarked ones (see note 13), but only that there is the same sort of a priori reason to assume that the simplest case is also a real possibility. We return to this point below.
} 
content of the BV is unclear in similar ways, for reasons discussed in the previous section. With these provisos in mind, I will briefly look at two types of phenomena that would play a crucial role in any event.

The first point is the position of the verbal head of simple clauses. A s mentioned above, $K \& P$ seem to make the principled claim that verb-second is a fundamental property of BV syntax. A Ithough this follows only implicitly from their phrasal constraints, there are at least no indications of any other options for (finite) verb placement. ${ }^{21} A$ ccording to $K \& P^{\prime}$ 's basic claim, verb second would also be the effect if all formal features $C_{H L}$ has access to were weak. Whether this conjecture is borne out is difficult to assess. A ctual analyses of e.g.verb second in E nglish or F rench consider the overt position of the verb as anything but a simple case with no overt movement. ${ }^{22}$ There are in fact many proposals where the assumption of only weak features would result in prefinal rather than second position of the verb. A case in point would be $\mathrm{H}$ ale and Keyser's (1993) account of causative verbs.

Pending further clarification, we might tentatively assume that verb second might be the effect of the smallest number of strong features $C_{H L}$ could live on. Whether this assumption will eventually be different from the position advocated by $K \& P$ depends on clarification within the $M$ inimalist Program. $M$ y hunch is that there will be a difference (for reasons mentioned in note 20).

The next point to be considered is the theory of thematic roles, and more specifically the principle SE M 1, which - as we saw excludes so-called psych-verb constructions like he/it pleases me. A ccording to the $M$ inimalist Program, the properties distinguishing pairs like please and like must be encoded in their lexical representation, such that $\mathrm{C}_{\mathrm{HL}}$ will then operate accordingly. O nce again, the details handling these properties are not quite obvious (but see Bierwisch, 1997, for some considerations). A necessary assumption seems to be that formal features involved in relating the verb to its arguments must somehow be connected to the conditions the verb imposes on the conceptual interpretation. In any case, the assignment of thematic roles to syntactic constituents must be determined by lexical properties - whether couched in terms of Control or any other terminology. G iven these preliminary

\footnotetext{
${ }^{21}$ See note 1 for one apparent exception. Notice that optional omission of the subject would not be in conflict with the generalization under discussion.

${ }^{22} \mathrm{O} n$ the analysis given in Wilder (1995) - and in fact much earlier work - verb second in German would after all be the more complex case, since at least one more instance of $M$ ove triggered by a strong feature is required than in the less complex verb-final constructions. $B$ ut this, of course, bears (at most) indirectly on the issue at hand.
} 
clarifications, principle SE M 1 would correspond to the assumption that features characterizing the relevant property of verbs like please are strong and hence not available in the BV. I don't think that this is the appropriate account for problems of thematic role assignment, for reasons that I would like to elaborate in a slightly more general way.

It seems to me an indispensable assumption that the theory of U G must provide among others a characterization of possible data structures that can become members of LS. Technically speaking, U G must contain a characterization of the notion 'possible lexical entry'. Both the M inimalist Program and the Basic Variety are at best incomplete in this respect, even though both recognize the importance of lexical information as the point of departure for all combinatorial operations that create the expressions of Ilanguage. ${ }^{23}$ It is the organization of lexical information that must support the principles of thematic roles and the way in which they can be projected on to the constituents of complex expressions. U nder this perspective, SE M 1 to SE M 3 - or rather the constraints that should replace them in the sense discussed earlier - should be derivable from the constraints on possible lexical information.A nd the particular role of the so-called psych(ological) verbs like please, strike, etc. would then be a matter of (more marked?) lexical entries, instead of the strong/weak distinction of formal features.

R eturning to the main point, neither verb placement nor thematic role assignment yields clear evidence with respect to the claim that the $B V$ is $C_{H L}$ with only weak formal features. Discussion of functional categories, topic-focus-articulation, Case theory, and several other issues would show that this is apparently not an incidental conclusion concerning only marginal phenomena. It rather reflects a lack of clarity on both sides, including the present state of the Minimalist Program. Hence for me the question in which way the generalizations embodied in the BV square with the theory of $U G$, especially its version of the M inimalist Program, remains unsettled.

That is not all there is to say, however. A nd this brings me to some more general remarks on the relation between the BV and U G. To this effect, I want to emphasize two observations that have influenced the concern for $U G$ in generative linguistics over the years.

First, language has been considered as a biologically fixed

\footnotetext{
${ }^{23}$ It might be noted that one important aspect of this problem has been dealt with (e.g., in Chomsky, 1981) with respect to so-called whether-verbs. The issue of 'impossible verbs' is also taken up in Chomsky (1995: 313). A more general discussion is nevertheless still pending.
} 
capacity (presumably species-specific) of human beings. More specifically, UG has been considered as the genetically fixed endowment that makes language acquisition possible and accounts for the solution of what has been called the logical problem of language acquisition. See Chomsky (1986) for discussion of 'Plato's Problem' and the proposal that UG accounts for the particular conditions involved in language acquisition.

Second, while there clearly must be some sort of innate basis for the language capacity, its specificity has been a matter of considerable discussion and rethinking. I nitially, the assumption of a strictly domain-specific basis relying on irreducible, autonomous principles seemed to be indispensable. ${ }^{24}$ The orientation connected to the Minimalist Program, which tries to be as parsimonious as possible with respect to irreducible principles, has obviously changed this orientation in one crucial respect. The particular properties of $\mathrm{C}_{\mathrm{HL}}$ and the I-language based on it are largely derived from general principles of cognitive organization and conceptual parsimony. The main feature of language seems to be the availability of discrete infinity, i.e. recursivity of computation. I don't want to enter the interesting discussion concerning especially the evolution of the language capacity this change of orientation has created. ${ }^{25}$ I want to stress two points, though. First, the orientation and, to some extent, the results of the Minimalist Program provide a cognitive foundation of the language capacity that one could describe as relativized autonomy. ${ }^{26}$ Second, this does not obviate the original insight that the language capacity and the processes of language acquisition and use it determines are biologically fixed properties of the human mind.

With this more general perspective on UG - which I take to include conditions on lexical organization, as emphasized above I want to return to the issue how $U G$ and the $B V$ might be related. For the sake of argument, I will assume that the BV can in fact eventually be characterized as a type of I-language that relies on

\footnotetext{
${ }^{24} \mathrm{~A}$ particularly strict version of this approach was Fodor's (1983) notion of modularity, according to which language functions as a reflex-like, encapsulated and strictly autonomous input-output system. The notion of modularity advocated, for example, in Chomsky (1986) is less rigid,but still keeping to the assumption that I-language is based on specific principles of its own.

${ }^{25}$ See, e.g., Pinker (1994) and Bickerton (1995) for different approaches to the phylogenetic aspect of language.

${ }^{26}$ This should not be construed as a poor compromise. I take the autonomy to be determined by (i) the recursivity just mentioned,and (ii) the correspondence between the independently given domains A -P and C-I, as shown in (15). See Bierwisch (1997) and references given there for some discussion. The autonomy is relativized, on the other hand, as both (i) and (ii) draw on cognitive organization in general, and might phylogenetically be explained as emergent properties of the growing brain.
} 
largely unspecific ways to relate patterns of A - $\mathrm{P}$ to structures in $\mathrm{C}$ I. By 'largely unspecific' I do not mean weak or unmarked in the technical sense, but merely as recruiting generally available cognitive resources. A s this perspective seems to me in line with much of what $K \& P$ want to defend, but still different in crucial respects, I want to elaborate somewhat the view I have in mind.

Notice, first of all, that the type of spontaneous $L 2$ acquisition $K \& P$ are concerned with differs from basic language acquisition in two important ways: first, adults have fully developed skills and representations in the domain of $\mathrm{C}-\mathrm{I}$; second they went through the normal process of $L 1$ acquisition, with the respective results. $B$ oth conditions distinguish $L 2$ from $L 1$ acquisition. This is, of course, commonplace, but needs to be emphasized. B oth conditions might be an advantage or a handicap for the $L 2$ learner. In any case, the initial state of the process of $L 2$ acquisition is quite different from that of $L 1$ acquisition. N ormal conditions of $L 1$ acquisition not only require children to build up their conceptual system in terms of which linguistic expressions get interpreted, they might also provide for cerebral plasticity supporting, e.g., complex morphology or intricate patterns of movement, no longer operative at the stage of L 2 acquisition.A nyway, L 2 learners will rely on different cognitive resources compared to children during $L 1$ acquisition.

This is not a new philosophy, but it seems to me very much in line with most of the observation $K \& P$ include in their $B V:$ general cognitive strategies of linearization both within and between clauses, very little overt grammatical marking, organization of lexical knowledge by resort to already acquired knowledge wherever this is compatible with actual needs.

If this is correct, then the BV does not depend on U G in the way in which initial I-language is based on and controlled by $U G$. $E$ xactly to the extent to which the BV is a variety on its own, not to be judged from the target $L 2$ - a crucial point in $K \& P^{\prime} s$ perspective - it is based on general cognitive strategies, rather than on U G. The similarity of the BV as some sort of a stable state to regular I-language would result from the fact that $U G$ to a large extent is based on general principles of cognitive organization. The difference of the BV to ordinary I-language, however, derives from the biologically determined specificity of the language capacity with conditions on ontogenetic development which simply do not obtain under adult $\mathrm{L} 2$ learning.

I want to conclude these somewhat speculative remarks by a fairly uncontroversial observation that clearly shows that the BV must not be construed as U G with only weak formal features, where $K \& P$ relate this property to the morphological poverty of the $B V$. 
A ccording to $K \& P^{\prime} s$ construal of the $B V$ as a natural result determined by $U G$, on a par with other I-languages, differing only by the condition that the BV is more perfect than ordinary Ilanguages, because the BV permits only weak formal features, one should expect natural languages in general to show a strong tendency towards the BV. To put it differently, if strong features are expensive, and hence avoided by $L 2$ learners, there is no reason why this tendency to avoid strong features should not be equally effective in $L 1$ acquisition, and hence a driving force in language change. Nothing like that is actually the case, of course. This must be taken as a clear indication that the $\mathrm{BV}$ is not a simplified version of normal I-language, but rather the result of a process that is qualitatively different from that of fixing strong and weak, or marked and unmarked, values of formal, semantic or phonetic features during first language acquisition.

To sum up: although the notion of the $B V$ provides an interesting perspective on spontaneous $L 2$ acquisition and its relation to $U G$, I do not think that it can be put in the same domain as ordinary Ilanguage, independently of ways to overcome some of the shortcomings discussed above. O ne need not consider the BV as merely a defective variety of a canonical target language; but it clearly is not a more perfect variety either. Complex morphology, marked phonetic features, complex patterns of word order, differences in thematic structure, and all the rest not occurring in the BV, must not be taken as just so many accidents in I-language construction, but rather as normal options within a process that is crucially different from BV acquisition.

To put it the other way round, and in a more positive guise: the concise characterization of general patterns on which $L 2$ learners converge can be taken as indicating that spontaneous $L 1$ acquisition based on language-specific conditions of $U G$ is essentially different from the processes of $L 2$ acquisition that exploit general cognitive strategies. U nder this perspective, the BV would come out as an interesting hypothesis concerning the difference between two different ways acquiring linguistic knowledge. A lthough this is clearly not what $K \& P$ wanted to establish, it would be a non-trivial and rather important result.

\section{R eferences}

B ickerton, D. 1995: L anguage and human behavior. Seattle: U niversity of Washington Press.

Bierwisch, M. 1997: Lexical information from a minimalist point of view. In Wilder, C., G ärtner, H.-M . and B ierwisch, M., editors, The role of 
economy principles in linguistic theory, Berlin: A kademie Verlag, 227-66.

Chomsky, N. 1965: A spects of the theory of syntax, Cambridge, M A : M IT Press.

---- 1981: L ectures on Government and Binding. D ordrecht: Foris.

---- 1986: K nowledge of language: its nature, origin and use. New York: Praeger.

---- 1995: The M inimalist Program. Cambridge, M A : M IT Press.

Comrie, B. 1981: L anguage universals and linguistic typology. Chicago: U niversity of Chicago Press.

D owty, D. 1991: Thematic proto roles and argument selection. L anguage 67, 547-619.

Fodor, J .A . 1983: The modularity of mind. Cambridge, M A : M IT Press.

G ärtner, H .-M. 1997: Generalized transformations and beyond. D issertation, H umboldt-U niversität B erlin.

G rimshaw, J. 1990: A rgument structure. Cambridge, M A : M IT Press.

Hale, K. and Keyser, S.J. 1993: O n argument structure and the lexical expression of syntactic relations. In $\mathrm{H}$ ale, $\mathrm{K}$. and $\mathrm{K}$ eyser, S.J ., editors, The view from building 20, Cambridge, M A : M IT Press, 53- 109.

Jackendoff, R. 1990: Semantic structures. Cambridge, M A : M IT Press.

Pinker, S. 1994: The language instinct. N ew Y ork: William M orrow.

Wilder, C. 1995: D erivational economy and the analysis of V 2. FA S Papers in $L$ inguistics $1,117-56$. 